\title{
A silver impregnation method for motor and sensory nerves and their endings in formalin-fixed mammalian muscles
}

\author{
(Received 23 October 1987) \\ (Revised 29 February 1988) \\ (Accepted 4 March 1988)
}

Hermann Schweizer and Heike Kaupenjohann

Zoologisches Institut der Ludwig-Maximilians Universität, Munich (F.R.G.)

Key words: Motor nerve ending; Sensory nerve ending; Motor end plate; Muscle spindle; Peripheral nervous system; Silver impregnation

\begin{abstract}
A silver impregnation method is described which shows motor and sensory nerves and their endings in formalin-fixed mammalian muscles. The method works with the same reliability on flattened muscle pieces as well as on frozen sections. Large nerve bundles, myelinated and non-myelinated single axons, and terminals impregnated by this method stand out black against a light brown background.
\end{abstract}

\section{Introduction}

Several histological methods are currently used to study motor and sensory innervation of mammalian muscles. Some of these procedures are performed on muscle pieces by block impregnation with silver salt solutions followed by teasing apart the impregnated tissue; others are performed on histological sections.

The block impregnation techniques have the advantage that it is possible to visualize more or less completely the innervation pattern of entire muscle fibers and muscle spindles, and that muscle fibers, and especially muscle spindles, can be isolated for further histological investigation.

The most commonly used methods are the silver impregnation method of Barker and Ip (1963) with its more recent modification (Barker et al., 1985) or the method of Gladden (1970) for demonstrating the motor and sensory innervation of

Correspondence: H. Schweizer, Zoologisches Institut der Ludwig-Maximilians Universität, Luisenstrasse 14, D-8000 München 2. F.R.G. muscle fibers, muscle spindles and tendon organs. These methods are mainly designed for use on muscle blocks. On histological sections different reduced silver methods are used (Namba et al., 1967; Hopkins, 1981; Cruz et al., 1984). However, the block techniques are not suitable for histological sections, and vice versa.

It is our experience that the block techniques give unsatisfactory results on small muscles thinner than about $1 \mathrm{~mm}$ because the muscle surface becomes too darkly impregnated.

In studies on the facial musculature in small mammals we tried to develop a method which can be used interchangeably on small whole muscles, muscle blocks and histological sections. In this method, whole muscles and blocks are flattened prior to impregnation in order to preserve the advantage of the block techniques, namely the visualization of the entire innervation pattern.

\section{Procedure}

Animals are deeply anesthetized (we use pentobarbital) and perfused through the heart with physiological saline followed by $10 \%$ formalin in 
unbuffered saline for $20 \mathrm{~min}$. Small muscle pieces or complete muscles (maximum $1 \mathrm{~mm}$ thick) are removed and immediately flattened by pressing them between two slides one of which is coated with gelatine chrome-alum (1.5 g gelatine are dissolved in $250 \mathrm{ml}$ distilled water, $0.08 \mathrm{~g}$ potassium chrome-alum are added. Slides are dipped into this solution air dried and dipped and dried again). After pressing the tissue to an appropriate thickness the two slides are fastened together by two plastic clamps. These slide sandwiches are put into a jar containing $10 \%$ unbuffered formalin for $24 \mathrm{~h}$ (overnight). If sections are to be prepared instead of a flattened block the tissue is cut on a freezing microtome at $30-100 \mu \mathrm{m}$ and the sections are mounted on gelatine chrome-alum coated slides. Thick sections should be mounted between two slides as described for the flattened preparations. After $24 \mathrm{~h}$ (the next day) the slides are separated from one another. By this procedure the tissue sticks firmly to the coated slide during all following steps. The described impregnation method can also be used on unmounted flattened muscle tissue if both of the slides used during the flattening procedure are uncoated. Mounted or unmounted tissue should be postfixed in $10 \%$ unbuffered formalin for an additional 4-5 days. After fixation the tissue is washed in several changes of distilled water for $24 \mathrm{~h}$ and then transferred to alcoholic ammonia (100 $\mathrm{ml} 96 \%$ ethanol $+1.5 \mathrm{ml}$ ammonia $25 \%$ ) for $48 \mathrm{~h}$. The jar used during this step should be tightly covered to prevent evaporation of the ammonia. Tissue is washed in 3 changes of distilled water at $10 \mathrm{~min}$ each, and incubated in the dark for $90 \mathrm{~min}$ in $0.75 \%$ silver nitrate. Plastic or glass instruments should be used to transfer slides or tissue. The solution should be agitated during this and the following steps (we use a magnetic stirrer). After rinsing in three changes of distilled water at $1 \mathrm{~min}$ each, the tissue is transferred to a modified Gallyas light insensitive physical developer (Gallyas, 1979) for 3-7 minutes.

This developer consists of a mixture of 3 stock solutions which are stable at room temperature for several weeks and are mixed together immediately prior to use.

Solution A: 50 g sodium carbonate (anhydrous) in $1000 \mathrm{ml}$ distilled water. Solution B: in the following order: $2 \mathrm{~g}$ ammonium nitrate, $2 \mathrm{~g}$ silver nitrate, $10 \mathrm{~g}$ tungstosilicic acid in $1000 \mathrm{ml}$ distilled water. Solution C: same composition as solution B plus $3.5 \mathrm{ml}$ formalin (37\% formaldehyde). At room temperature $\left(18-25^{\circ} \mathrm{C}\right)$ the working solution is made by mixing 3 parts A plus 2 parts $B$ plus 1 part $C$ in this order under vigorous stirring. The final solution is stable for about $20 \mathrm{~min}$ and can be used only once. We did not change the mixing ratio of the developer according to the temperature as suggested by Gallyas, but at higher or lower temperatures than normal, this may be necessary (more of solution $\mathrm{B}$, and correspondingly less of solution $C$, and vice versa). For more detail see Gallyas (1979).

Although only two stock solutions would be necessary to prepare the developer under normal conditions, the use of 3 stock solutions is more convenient if it is necessary to change the mixing ratio according to the temperature or to adapt the method to other tissues. In the case of two stock solutions, solution $\mathrm{C}$ is omitted, and $1.2 \mathrm{ml}$ formalin is added to solution $B$. The mixing ratio is then 1 part A plus 1 part B.

During development sections should be agitated and the development must be monitored under the microscope. When development is sufficient, the process can be stopped by transferring the sections to a solution of $0.5 \%$ acetic acid. Sections are then rinsed in distilled water and fixed in $2.5 \%$ sodium thiosulfate for $2 \mathrm{~min}$. They are then washed in running tap water for $15-30 \mathrm{~min}$, rinsed in distilled water, dehydrated in a graded series of alcohols, cleared in xylene and mounted in Depex or a similar mounting medium (the medium should be tested for compatibility with silver impregnation). The tissue can also be mounted in glycerine or polyvinyllactophenol without dehydration.

\section{Results}

In all material tested (various limb, facial, masticatory and neck muscles from mice and bats) the described silver method proved to be a good and reliable means of demonstrating muscle innervation in both sections and flattened preparations. 

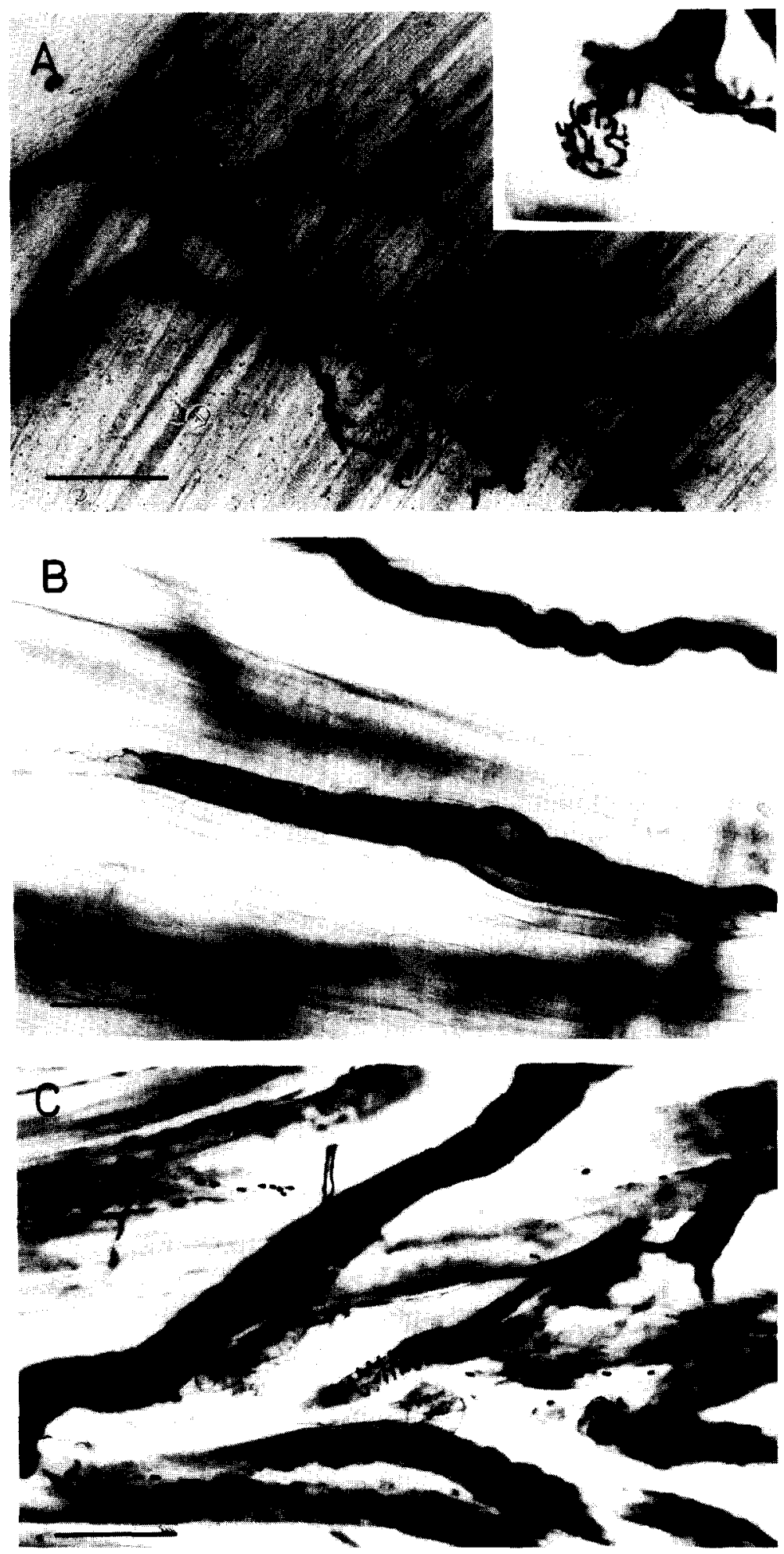

Fig. 1. Examples of impregnated nerve fibers. A: motor nerve splitting off and terminating in motor end plates on muscle fibers (bat neck muscle). Note that the quality of impregnation is equally good for nerve bundles, single fibers and terminals. Inset in $\mathrm{A}$ : a motor end plate at higher magnification. B: impregnation of a muscle spindle. Note the sharpness with which impregnation of fine fibers can be visualized. C: primary endings of sensory spindle fibers (frozen section). B and C are preparations from mouse hindlimb muscles. The calibration bar represents $110 \mu \mathrm{m}$ in A, and B, $80 \mu \mathrm{m}$ in C. and $50 \mu \mathrm{m}$ for the inset in A. 
The black or dark brown nerve fibers and nerve terminals stand out in high contrast against a nearly colorless or lightly brown background of muscle fibers. Even in slightly overdeveloped tissue there is sufficient contrast to differentiate between muscle and nerve fibers. The only nonneuronal structures that are impregnated in muscle tissue are the nuclei of muscle fibers, red blood cells, and occasionally the walls of blood vessels. Fatty and connective tissue are also impregnated, but even in these tissues neural elements are clearly recognizable by their darker staining. The quality of impregnation is equally good for large and small nerve fiber bundles, thick and thin single fibers, and motor and sensory nerve terminals.

\section{Discussion and Remarks}

The above method is an effective means of demonstrating nerve fibers and nerve endings in mammalian muscles. In contrast to the method of Barker and Ip (1963) which gives beautiful impregnations on muscle blocks and small whole muscles our method can be used on flattened muscle pieces, small whole muscles, and frozen sections from formalin-fixed material. For the use on frozen sections our method is easier to handle than comparable methods (e.g. Namba et al., 1967; Cruz et al., 1984). The method apparently impregnates all nerve fibers within a given muscle block, with incomplete staining only if the tissue is too thick. Although we did not test the method on other mammals it seems reasonable to assume that it works on all tissues or can easily be adapted by changing the concentration of ammonia (higher concentration), by shortening or prolonging the impregnation time, or by changing the mixing ratio of the developer. Furthermore, it may be possible that the method also works on tissue which has been fixed for more or less than the tested 5 days.

\section{Acknowledgements}

We thank Drs. G. Neuweiler and E. Covey for critical reading of the manuscript and Mrs. $H$. Tscharntke for photographical work. Supported by the Deutsche Forschungsgemeinschaft (DFG), Sonderforschungsbereich 204 "Gehör", TP 14.

\section{References}

Barker, D. and Ip, M.C. (1963) A silver method for demonstrating the innervation of mammalian muscle in teased preparations, J. Physiol. Lond., 169: 73-74P

Barker, D., Scott, J.J.A. and Stacey, M.J., (1985) Sensory reinnervation of cat peroneus brevis muscle spindles after nerve crush, Brain Res., 333: 131-138.

Cruz, M.C., Jeanmonod, D., Meier, K. and Van der Loos, H. (1984) A silver and gold technique for axons and axon-bundles in formalin-fixed central and peripheral nervous tissue, J. Neurosci. Methods, 10: 1-8.

Gallyas, F. (1979) Silver staining of myelin by means of a physical development, Neurol. Res., 1: 203-209.

Gladden, M.H. (1970) A modified pyridine-silver stain for teased preparation of motor and sensory nerve endings in skeletal muscle, Stain Technol., 45: 161-164.

Hopkins, W.G. (1981) Age-related changes in innervation of normal and paralysed mouse muscles studied with a simplified silver stain, J. Physiol. (Lond.), 320: 5P

Namba, T., Nakamura, T. and Grob, D. (1967) Staining for nerve fiber and acetylcholinesterase activity in fresh frozen sections. Am. J. Clin. Path. 47: 74-77. 